\title{
Nuevos registros de líquenes de Quintana Roo, México
}

\author{
(New lichen records from Quintana Roo, Mexico) \\ Guzmán-Guillermo, J. ${ }^{1 *}$, Cárdenas-Mendoza, K.D.R. ${ }^{1}$, Huereca, A. ${ }^{2}$ \\ ${ }^{1}$ Facultad de Biología, campus Xalapa, Universidad Veracruzana. \\ Veracruz, México. \\ ${ }^{2}$ Facultad de Ciencias Biológicas, Universidad Autónoma \\ de Nuevo León. San Nicolás de los Garza, México \\ *Autor para correspondencia: qwerty.guzmi@gmail.com
}

RECIBIDO: 06 de Septiembre de 2019

APROBADO: 02 de Diciembre de 2019

DOI: 10.22370/bolmicol.2019.34.2.1903

LOS AUTORES DECLARAN NO TENER CONFLICTO DE INTERESES

Palabras claves: microliquenes; Graphidaceae; Arthoniaceae; nuevos registros.

Key words: microlichens; Graphidaceae; Arthoniaceae; new records.

\section{RESUMEN}

En el presente trabajo se describen ocho especies de líquenes crustáceos (Arthonia cinnabarina (DC.) Wallr., Arthothelium macrothecum (Fée) A. Massal, Bactrospora jenikii (Vezda) Egea \& Torrente, Cresponea proximata (Nyl.) Egea \& Torrente, Dirina paradoxa (Fée) Tehler, Graphis glaucescens Fée y Sarcographa tricosa (Ach.) Müll. Arg.) y una especie foliosa (Pyxine cocoës (Sw.) Nyl.) como nuevos registros para el estado de Quintana Roo y Graphis subchrysocarpa Lücking como nuevo reporte para México.

\footnotetext{
ABSTRACT

In the present study, we found eight crustose lichens (Arthonia cinnabarina (DC.) Wallr., Arthothelium macrothecum (Fée) A. Massal, Bactrospora jenikii (Vezda) Egea \& Torrente, Cresponea proximata (Nyl.) Egea \& Torrente, Dirina
}

paradoxa (Fée) Tehler, Graphis glaucescens Fée and Sarcographa tricosa (Ach.) Müll. Arg.), all of them are reported as new for Quintana Roo and Graphis subchrysocarpa Lücking as a new record for Mexico.

\section{INTRODUCCION}

Se estima que la diversidad de líquenes en México es elevada, así como se ha demostrado para otros grupos de hongos. Sin embargo, los estudios liquenológicos en el país son aún escasos y se concentran en unos pocos estados (HerreraCampos et al., 2014). Para Quintana Roo no existe ningún trabajo que haya abordado líquenes de manera exclusiva. Sin embargo, se sabe que hay 80 especies reportadas para ese estado (HerreraCampos et al., 2014). En el presente trabajo se describen ocho especies corticícolas recolectadas en distintas localidades del estado de Quintana Roo, México. 


\section{MATERIALES Y MÉTODOS}

Se recolectaron muestras liquénicas en los municipios de Bacalar y Othon P. Blanco, en el estado de Quintana Roo. De 60 muestras recolectadas, se determinaron 40 ejemplares hasta especie, mediante las técnicas convencionales de liquenología detalladas por Brodo et al. (2001), y se observaron y midieron las estructuras microscópicas sexuales con un microscopio modelo Primo Star marca Carl Zeiss y estructuras macroscópicas con un microscopio estereoscópico modelo Stemi Dv4 marca Carl Zeiss en el Laboratorio de Calidad Ambiental de la Facultad de Biología de la Universidad Veracruzana. En el caso de las estructuras microscópicas aquí descritas, se midieron 30 elementos (esporas, en algunos casos ascas y parafisas) de cada ejemplar y se promediaron las medidas. Se utilizaron las siguientes claves taxonómicas en la identificación de los ejemplares estudiados: Aptroot et al. (2014); Brodo et al. (2001); Bungartz et al. (2010); Egea y Torrente (1993A, 1993B); Lucking et al. (2008, 2009); Nayacka y Upreti, 2005; Sipman et al. (2012); Sobreira et al. (2015); Tehler et al. (2013). Posteriormente, la distribución geográfica de estas especies en México fue determinada utilizando trabajos previos (Egea y Torrente, 1993A, 1993B; Herrera-Campos et al., 2017; Sipman y Wolf, 1998; Tehler et al., 2013) y la base de datos del Consorcio Norteamericano de Herbarios de Líquenes (CNALH).

\section{RESULTADOS}

Se determinaron ocho especies de líquenes crustáceos y una foliosa, de las cuales ocho son nuevos registros para Quintana Roo y uno para México. La mayor parte de las especies determinadas corresponde al orden Arthoniales (con tres familias

Tabla 1. Información taxonómica de las especies encontradas.

\begin{tabular}{|c|c|c|}
\hline Orden & Familia & Nombre científico \\
\hline \multirow[t]{4}{*}{ Arthoniales } & \multirow[t]{2}{*}{ Arthoniaceae } & Arthothelium macrothecum (Fée) A. Massal \\
\hline & & Arthonia cinnabarina (DC.) Wallr. \\
\hline & Opegraphaceae & Cresponea proximata (Nyl.) Egea \& Torrente \\
\hline & Roccellaceae & Dirina paradoxa (Fée) Tehler \\
\hline Caliciales & Caliciaceae & Pyxine cocoës (Sw.) Nyl. \\
\hline Incertae sedis & Incertae sedis & Bactrospora jenikii (Vezda) Egea \& Torrente \\
\hline \multirow[t]{3}{*}{ Ostropales } & \multirow[t]{3}{*}{ Graphidaceae } & Sarcographa tricosa (Ach.) Müll. Arg. \\
\hline & & Graphis glaucescens Fée \\
\hline & & Graphis subchrysocarpa Lücking \\
\hline
\end{tabular}


y cuatro especies), seguido de Ostropales (con una familia y tres especies) y finalmente una especie de Caliciaceae y una Incertae sedis (Tabla 1). A continuación se hace la descripción de las especies encontradas, agregando información sobre su distribución en México.

\section{Arthothelium macrothecum (Fée) A. Massal}

Talo crustáceo blanquecino, liso, ecorticado, areolado. Presencia de una línea divisoria derivada del hipotalo color negra que delimita el talo. Apotecios circulares a estrellados, inmersos planos, no estromáticos, exponiendo solamente el himenio marrón oscuro. Ascos octospóricos, globuliformes. Ascosporas muriformes con hasta 30 lóculos y la parte media más delgada, de 55-60 x $17 \mu \mathrm{m}$. Reacciones: ascos I+ azul; talo K-, C-. Figura: 1C.

Notas: Se diferencia de otras especies con esporas muriformes y con un margen negro derivado del hipotalo como A. spectabile, debido a que sus esporas presentan un tamaño de 25-30 $\mu \mathrm{m}$ de largo (Sudin y Tehler, 1998), lo cual es considerablemente menor a lo documentado para $A$. macrothecum (Nayaca y Upreti, 2005).

Distribución: Para México esta especie ha sido reportada por Herrera-Campos et al. (2017) para el estado de Jalisco.

Material estudiado: QUINTANA ROO, Municipio de Othon P. Blanco, entrada a la Zona Arqueológica de Kohunlich, Diciembre 27, 2016. GuzmánGuillermo, 379-A.

\section{Arthonia cinnabarina (DC.) Wallr.}

Talo crustáceo, blanquecino a ligeramente verdoso cuando húmedo, ligeramente areolado. Apotecios discoideos, sésiles con un margen cubierto por pruina roja, epitecio blanco. Ascas octospóricas, clavadas. Esporas fusiformes con un extremo más ancho, hialinas con 4 septos y 5 lóculos, de 20-22 x 7-9 $\mu \mathrm{m}$. Reacciones: pruina roja $\mathrm{K}+$ púrpura; talo K-, C-, I-. Figura: 1E.

Notas: características como el tamaño y forma de las esporas y presencia de pruina $\mathrm{K}+$ purpura en el margen del apotecio coinciden con lo reportado por Sudin y Tehler (1998).

Distribución: esta especie fue reportada previamente para los estados de Chiapas (Sipman y Wolf, 1998), Jalisco (Herrera-Campos et al., 2017), Michoacán, Nuevo León, Oaxaca y Tamaulipas (CNALH).

Material estudiado: QUINTANA ROO, Municipio de Othon P. Blanco, entrada a la Zona Arqueológica de Kohunlich. Diciembre 27, 2016. GuzmánGuillermo, 382., Comunidad de Obregón Nuevo casa particular del maestro E. Borges. Diciembre 29, 2016. Guzmán-Guillermo, 451, 452.

\section{Bactrospora jenikii (Vezda) Egea \& Torrente}

Talo crustáceo blanquecino a grisáceo, ecorticado. Apotecios discoideos, negros, sésiles, con los márgenes arrugados, de aspecto verrucoso, excípulo carbonizado. Ascos clavados de 84-87 x 8-11 $\mu \mathrm{m}$. Parafisas ramificadas hacia las puntas, de $100 \times 2 \mu \mathrm{m}$. Ascosporas aciculares con una ligera constricción hacia la parte media, con 7-11 septos y 8-12 lóculos, de 36-46 x $2 \mu \mathrm{m}$. Reacciones: K-, C-, I-. Figura: 1A.

Notas: Se puede diferenciar de otras especies como $B$. incana, B. brevispora, en el número de septos, por otro lado $B$. jenikii ha sido previamente citada para el Caribe (Sobreira et al., 2015).

Distribución: el género ha sido reportado para México en los estados de Guerrero y Nayarit (CNALH) y Jalisco (Herrera-Campos et al., 2017), y la especie para el estado de Nayarit (CNALH).

Material estudiado: QUINTANA ROO, Municipio de Bacalar, Balneario de los cañeros en la Laguna de Bacalar. Diciembre 26, 2016. GuzmánGuillermo, 427, 428, 429.

\section{Cresponea proximata (Nyl.) Egea \& Torrente}

Talo crustáceo, color amarillento, estriado, con un hipotalo marcando una línea café oscura a negra en el margen del talo. Apotecios discoideos numerosos, en todo el talo, sésiles, con la base constreñida, margen negro, epitecio cubierto por pruina 
amarillenta verdosa, hipotecio café. Ascas octospóricas, biseriadas. Ascosporas fusiformes con los lóculos centrales más anchos que los de los extremos, con 4-5 lóculos de 25-29 x 5-6 $\mu \mathrm{m}$. Reacciones: talo K-, C-; himenio I+ rojizo; hipotecio I+ azul. Figura: $1 \mathrm{H}$.

Notas: Las características diagnósticas para la especie, son el tamaño de las esporas, presencia de pruina amarillenta a verdosa sobre el epitecio y la presencia de una línea oscura derivada del hipotalo que delimita al talo (Egea y Torrente, 1993A).

Distribución: para México esta especie ha sido reportada para Jalisco (Herrera-Campos et al., 2017) y San Luis Potosí (CNALH).

Material estudiado: QUINTANA ROO, municipio de Bacalar, Balneario de los Cañeros en la Laguna de Bacalar. Diciembre 26, 2016. GuzmánGuillermo, 422, 423, 424, 425.

\section{Dirina paradoxa (Fée) Tehler}

Talo crustáceo corticado, color blanquecino a grisáceo verdoso cuando húmedo. Apotecios discoideos, centrípetos, margen talino blanco, sésiles con la base ligeramente constreñida, de 1.7-2.1 mm de diámetro, cubierto con pruina blanca, hipotecio negro evidentemente carbonizado. Ascas octospóricas, clavadas de $98 \times 12 \mu \mathrm{m}$. Ascosporas hialinas, fusiformes, con cuatro lóculos, de 30-37 x 5-6 $\mu \mathrm{m}$. Reacciones: talo $\mathrm{K}-, \mathrm{C}+$ rojo, pruina del disco $\mathrm{C}+$ rosa. Figura: $1 \mathrm{G}$.

Notas: las características reportadas por Tehler et al. (2013) para esta especie coinciden con las encontradas en estos ejemplares. Sin embargo, el tamaño de las esporas parece ser variable, este ejemplar muestra tamaños de hasta 37 × $6 \mu \mathrm{m}$, lo cual sobrepasaría por varios micrómetros la descripción antes mencionada. Esta especie puede encontrarse facultativamente en roca o en corteza, y sus esporas pueden alcanzar tales tamaños (Anders Tehler comunicación personal).

Distribución: esta especie ha sido reportada para Baja California, Baja California Sur y Oaxaca (CNALH). Tehler et al. (2013), mencionan que esta especie tiene distribución hacia la región del Caribe. Material estudiado: QUINTANA ROO, municipio de Bacalar, Balneario de los Cañeros en la Laguna de Bacalar. Diciembre 26, 2016. GuzmánGuillermo, 400, 401, 402, 403, 404, 405, 406, 407, $408,409,410,411,412,413,414,415,416,417$, $418,419,420$.

\section{Graphis glaucescens Fée}

Talo crustáceo ecorticado, color verdoso con coloraciones amarillentas, de aspecto estriado. Apotecios lireloides, de inmersos a ligeramente errumpentes, excípulo ligeramente carbonizado, cubiertos con pruina blanca. Ascas octospóricas, clavadas, de 98 x $20 \mu \mathrm{m}$. Ascosporas hialinas fusiformes con 9-12 lóculos (10 en promedio), de 33-40 x 8-9 $\mu \mathrm{m}$, parafisas no ramificadas, septadas de 115 x 1,5 $\mu \mathrm{m}$. Reacciones: Ascos I-; esporas I+ púrpura; talo K-, C-, I-. Figura: 1D.

Notas: Las características diagnósticas para esta especie fueron lirelas errumpentes, talo ecorticado, presencia de pruina blanca, excípulo ligeramente carbonizado, el número de septos, el tamaño de las esporas y reacciones negativas (Brodo et al., 2001; Lucking et al. 2008, 2009).

Distribución: para México se ha reportado en Jalisco (Herrera-Campos et al., 2017) y Chiapas (Sipman y Wolf, 1998).

Material estudiado: QUINTANA ROO, Municipio de Bacalar, Balneario de los Cañeros en la Laguna de Bacalar. Diciembre 26, 2016. GuzmánGuillermo, 426.

\section{Graphis subchrysocarpa Lücking}

Talo crustáceo ecorticado, blanquecino a verdoso cuando húmedo, ligeramente areolado. Apotecios lireloides, generalmente ramificados de manera irregular, márgenes carbonizados cubiertos con una pruina ocre a rojiza. Ascosporas transversalmente septadas con los extremos submuriformes, de 96-98 x $12 \mu \mathrm{m}$. Reacciones: esporas I+ púrpura; pruina $\mathrm{K}+$ púrpura intenso. Figura: $1 \mathrm{~F}$.

Notas: La especie G. subchrysocarpa se distingue 
de otras del grupo G. chrysocarpa por poseer esporas cuyos extremos son submuriformes (Lucking et al., 2008, 2009).

Distribución: G. subchrysocarpa no ha sido previamente citada para México. Tiene una distribución neotropical y ha sido citada para las costas Colombianas del Caribe por Rincón-Espita y Lücking (2011).

Material estudiado: QUINTANA ROO, Municipio de Othon P. Blanco, entrada a la Zona Arqueológica de Kohunlich, Diciembre 27, 2016. GuzmánGuillermo, 376.

\section{Pyxine cocoës (Sw.) Nyl.}

Talo folioso blanquecino a ligeramente verdoso cuando húmedo, con lóbulos irregularmente ramificados menores a $1 \mathrm{~mm}$ íntimamente adheridos al sustrato, corticado, sin estriaciones ni máculas. Médula blanca. Ausencia de cilios, presencia de pruina blanca en parches sobre el córtex superior. Córtex inferior negro. Presencia de soredios farinosos a granulares en el borde de los lóbulos hacia las partes centrales del talo. Reacciones: médula $\mathrm{K}+$ amarillo, C-; córtex K-, C-, UV+ amarillo. Figura: 1I.

Notas: Las características diagnósticas son la presencia de lichenxantona (UV+ amarillo intenso), soredios en los márgenes de los lóbulos y médula blanca. Difiere de P. katendei en la posición de los soredios (Aptroot et al. 2014).

Distribución: Previamente ha sido reportada para los estados de Jalisco (Herrera-Campos et al., 2017), Baja California Sur, Colima, Tamaulipas y Sonora (CNALH)

Material estudiado: QUINTANA ROO, Municipio de Othon P. Blanco, entrada a la Zona Arqueológica de Kohunlich. Diciembre 27, 2016. GuzmánGuillermo, 374, 386, 389, 393.

\section{Sarcographa tricosa (Ach.) Müll. Arg.}

Talo crustáceo ecorticado, color blanquecino a ligeramente verdoso cuando húmedo. Apotecios estromáticos lireloides inmersos, lirelas ramificadas irregularmente alcanzando $5 \mathrm{~mm}$ de diámetro, café oscuras, en ocasiones cubiertas con pruina blanca generalmente hacia las puntas, extremos redondeados, hipotecio carbonizado. Ascas octospóricas. Ascosporas fusiformes con tres septos, con cuatro lóculos, (17)19-21(22) x $7 \mu \mathrm{m}$. Reacciones: ascas I-; esporas I+ púrpura; talo K-, C-, I-. Figura: $1 \mathrm{~B}$. Notas: se diferencia de $S$. cinchonarum debido a que posee esporas con más de 4 septos. Otras características como la forma de los apotecios, tamaño y reacciones coinciden con lo reportado por Bungartz et al. (2010).

Distribución: esta especie se conoce de Jalisco (Herrera-Campos et al., 2017) y Chiapas (Sipman y Wolf, 1998).

Material estudiado: QUINTANA ROO, municipio de Bacalar, Balneario de los cañeros en la Laguna de Bacalar. Diciembre 26, 2016. GuzmánGuillermo, 430, 431. Municipio de Othon P. Blanco, entrada a la Zona Arqueológica de Kohunlich. Diciembre 27, 2016. Guzmán-Guillermo, 379-B.

\section{DISCUSIÓN}

Las especies determinadas han sido previamente reportadas en trabajos realizados en ecosistemas similares a la selva húmeda tal como los sitios de muestreo de este estudio (Bungartz, et al., 2010; Lucking et al., 2008; Rincón-Espitia y Lücking, 2011;). En el caso de Arthoniales se ha documentado que las especies de este orden son especialmente abundantes y diversas hacía las costas y las selvas, con características similares a las de Quintana Roo (Ertz et al., 2009).

Otro grupo con dominancia dentro de los microlíquenes es la familia Graphidaceae, en este estudio representaron la tercera parte de los organismos identificados. Según Lücking y Rivas-Plata (2008), las especies de esta familia son especialmente diversas y abundantes en las regiones tropicales desde el nivel del mar hasta aproximadamente los 2000 m.s.n.m. 

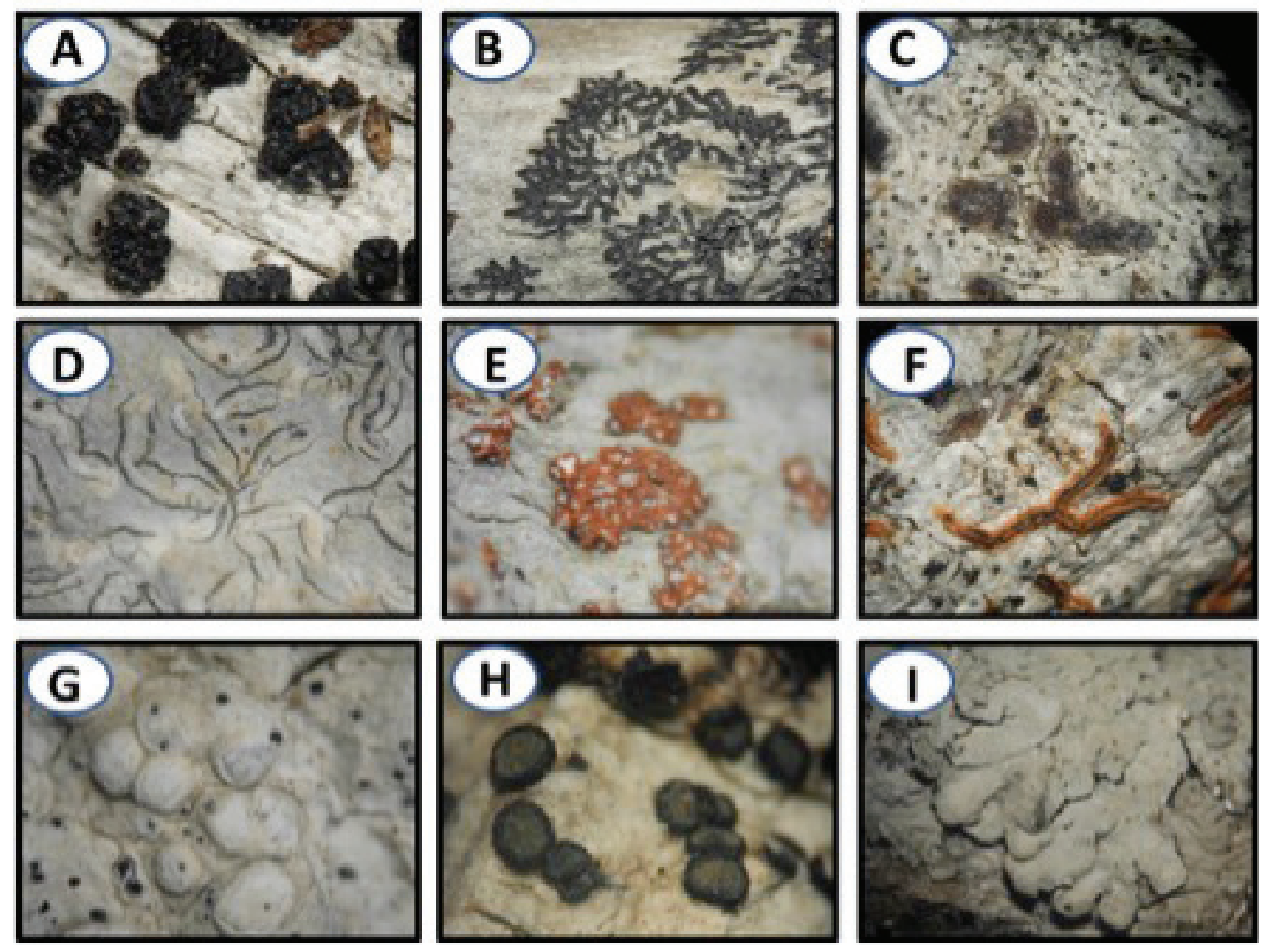

Figura 1. A. Bactrospora jenikii. B. Sarcographa trichosa. C. Arthothelium macrothecum. D. Graphis glaucescens. E. Arthonia cinnabarina. F. Graphis subchrysocarpa. G. Dirina paradoxa. H. Cresponea proximata. I. Pyxine cocoës.

Para México se sabe que el $62 \%$ de los líquenes conocidos corresponden a microlíquenes, y el tercer género más diverso es Graphis con 109 especies registradas (Herrera-Campos et al., 2014). Otros trabajos que han encontrado especies similares a las de este trabajo es el de HerreraCampos et al. (2017), realizado en una selva baja caducifolia (selva subhúmeda) en el estado de Jalisco, México.

\section{CONCLUSIONES}

El presente trabajo reporta 8 especies de líquenes no identificados anteriormente en el estado de Quintana Roo. Con las adiciones aquí presentadas a la funga liquénica, suman 88 especies conocidas para este estado. Los estudios liquenológicos en las costas del Mar Caribe y del Golfo de México deben continuarse.

\section{AGRADECIMIENTOS}

Agradecemos al Laboratorio de Calidad Ambiental de la Facultad de Biología de la Universidad Veracruzana por brindar acceso a sus microscopios y reactivos, al Herbario XALU en el procesamiento de los ejemplares aquí presentados, así como a los investigadores R. Lücking y A. Tehler por sus orientaciones en la determinación de estos organismos. De igual manera al maestro Borges por sus atenciones durante la recolección del material recolectado. 


\section{REFERENCIAS}

Aptroot, A., Jungbluth, P. y Cáceres, M.E.S. (2014). A world key to the species of Pyxine with lichexanthone, with a new species from Brazil. The Lichenologist, 45(5): 1-4, doi: 10.1017/ S0024282914000231

Brodo, I. M., Duran Sharnoff, S. y Sharnoff, S. (2001). Lichens of North America. Yale University Bungartz, F., Lücking, R. y Aptroot, A. (2010). The family Graphidaceae (Ostropales, Lecanoromycetes) in the Galapagos Islands. Nova Hedwigia, 90: 1-44, doi: 10.1127/0029-5035/2010/0090-0001

Egea, J.M. y Torrente, P. (1993A). Cresponea a new genus of lichenized fungi in the order Arthoniales (Ascomycotina). Mycotaxon, 48:301-331

Egea, J.M. y Torrente, P. (1993B). The lichen genus Bactrospora. Lichenologist, 25(3): 211-255, doi: 10.1006/lich.1993.1028

Ertz, D., Miadlikowska, J., Lutzoni, F., Dessein, S., Raspe, O., Vigneron, N., Hofstetter, V. y Diederich, P. (2009). Towards a new classification of the Arthoniales (Ascomycota) based on a three-gene phylogeny focussing on the genus Opegrapha. Mycological Research, 113(1): 141-152, doi: 10.1016/j.mycres.2008.09.002

Herrera-Campos, M. A., Miranda-González, R., Lücking, R., Sánchez-Téllez, N. y A. Barcenas-Peña. (2017). Inventario y base de datos de los líquenes de la selva seca de Jalisco. Universidad Nacional Autónoma de México. Instituto de Biología. Informe final SNIB-CONABIO, proyecto No. JF157. Ciudad de México

Herrera-Campos, M.A., Lücking, R., Perez-Perez, R.E., Miranda-Gonzales, R., Sanchez, N., Barcenas-Peña, A., Carrizos, A., Zambrano, A., Rhyan, B.D. y Nash, T.H. 2014. Diversidad de líquenes de México. Revista Mexicana de Biodiversidad, 85: 82-99, doi: 10.7550/rmb.37003
Lücking, R. y Rivas-Plata, E. (2008). Clave y Guía Ilustrada para Géneros de Graphidaceae. Glalia, 1: 1-41

Lücking, R., Archer, A.W. y Aptroot, A. (2009). A world-wide key to the genus Graphis (Ostropales: Graphidaceae). The Lichenologist, 41(4): 463452, doi, 10.1017/S0024282909008305

Lücking, R., Chaves, J.L., Sipman, H.J.M., Umaña, L. y Aptroot, A. (2008). A first assessment of the Ticolichen Biodiversity Inventory in Costa Rica: The genus Graphis, with notes on the genus Hemithecium (Ascomycota: Ostropales: Graphidaceae). Fieldiana Botany, 46:1-126, doi: 10.3158/0015-0746(2008)46[1:AFAOTT]2. $0 . \mathrm{CO} ; 2$

Nayaka, S. y Upreti, D.K. (2005). Status of Lichen Diversity in Western Ghats, India. Sahyadri E-News, Western Ghats Biodiverstity Information System - Issue XVI

Rincón-Espitia, A. y Lücking. (2011). New records of the genus Graphis (Graphidaceae) in Colombia. Bryophyte Diversity and Evolution, 33(1): 54-62, doi: 10.11646/bde.33.1.9

Sipman, H., Lücking, R., Aptroot, A., Chaves, J.L., Kalb. K. y Umaña T., L. (2012). A frst assessment of the Ticolichen biodiversity inventory in Costa Rica and adjacent areas: the thelotremoid Graphidaceae (Ascomycota: Ostropales). Phytotaxa, 55: 1-214. doi: 10.11646/phytotaxa.55.1.1

Sipman, H.J.M. y Wolf, J.H.D. (1998). Provisional checklist for the lichens of Chiapas. Acta Botánica Mexicana, 45:1-29, doi: 10.21829/ abm45.1998.808

Sudin, R. y Tehler, A. (1998). Phylogenetic studies of the genus Arthonia. Lichenologist, 30(4-5): 381-413, doi: 10.1017/S0024282992000409 
Tehler, A., Ertz, D. e Irestedt, M. (2013). The genus Dirina (Roccellaceae, Arthoniales) revisited. The Lichenologist, 45(4): 427-476, doi: 10.1017/ S0024282913000121 